\title{
Microstructure of replacement chickens' spleen after immunostimulant application
}

\author{
Rezilya Asrutdinova*, Rustem Mullahmetov, Lenar Rakhmatov, Nadezhda Kanalina and Salavat Garipov
}

Kazan State Academy of Veterinary Medicine named after N.E. Bauman, Kazan, 420029, Russia

\begin{abstract}
The article presents the histological studies of replacement chickens' spleen. The predominance of medium lymphocytes over small ones in the periarterial area was noted after vaccination with "Raspol" being an immunostimulant against chicken infectious bronchitis. In the periarterial area, the predominance of medium lymphocytes over small ones was noted. The lumen profiles of the central, trabecular arteries were well defined, and there were no signs of connective tissue disorganization in their walls. The marginal part of the lymph nodules also remained well defined especially from the inner border and consisted of a large number of small lymphocytes and fewer medium ones. However, its width decreased markedly compared to the previous period of the organ examination. The indicated changes denote the signs of lymphoid cell hyperplasia in the lymph nodes, with a weak severity or absence of the consequences of the vaccine reactogenic effect on the connective tissue of the organ. In the birds receiving Fosprenil during immunization, the stimulation of lymphoproliferative activity continued during the first two weeks after vaccination and by the end of the one-month period it was not detected. However, signs of moderate postvaccination disorganization of the connective tissue components were observed at all follow-up periods. 28 days after vaccination, lymphoproliferative processes weakened markedly and signs accompanied by moderate disorganization of the components in connective tissue persisted in the spleen of the control group of birds.
\end{abstract}

\section{Introduction}

Poultry farming is one of the most important sources of food resources replenishment. The products of this industry are distinguished not only by high consumer properties, but also by greater availability and affordability in comparison with other animal-derived products.

The basis of Russian poultry farming is large industrial-type poultry enterprises, where research work outcomes are being effectively implemented [1].

At present, Russian poultry farming is undoubtedly a leader in the market of livestock products. For more than half a century, the poultry industry has provided the country population with high-quality dietary products being poultry meat and eggs, whose production per capita has grown to $33 \mathrm{~kg}$ and $307 \mathrm{eggs}$, respectively [2].

In modern economic conditions, a successful industrial poultry farming can be achieved not only through the introduction of new technologies, augmenting a livestock with species and crosses of birds with high genetic potential and growth rate, but also through the use of new pharmacological developments and methods for improving the health of farm birds [3]. A judgment on the safety of immunomodulators is currently based on toxicity studies conducted on laboratory and other types of animals [4-9].
Under the conditions of industrial maintenance, with a high level of technological stress, secondary immunodeficiency states are recorded in almost $80 \%$ of the poultry stock [10]. Vaccination of poultry with a low level of resistance does not lead to the production of a sufficient amount of protective antibodies and is accompanied by an increase in the number of postvaccination complications.

Polysaccharides are considered as a promising complex of biologically active substances used for creating new drugs with the objective to eliminate various disorders of the immune system [11].

Taking the above into account, the search for new immunostimulating drugs of domestic production is an urgent task that requires their study, testing and implementation in veterinary practice.

The purpose of the work is to study the effect of plant heteropolysaccharidan on the microstructure of chickens' spleen when combined with the vaccine against chicken infectious bronchitis.

\section{Materials and Methods}

The effectiveness of heteropolysaccharide "Raspol" was determined by immunizing chickens against chicken infectious bronchitis with the SEVAC AyBerd vaccine. For this, 75 chickens of the egg direction of the

* Corresponding author: roza509a@ mail.ru 
"Lomann LSL" cross were used. They were divided into 3 groups, 25 chickens in each one. The birds of the first group were injected twice with "Raspol" with an interval of 3 days at a dose of $133.2 \mathrm{mg} / \mathrm{g}$. The first injection was made on the day of vaccination, the second one was given 3 days after immunization. The second group was injected with Fosprenil at the dose of $0.05 \mathrm{ml} / \mathrm{kg}$ according to the same scheme. Control group was represented by 25 birds vaccinated without an immunostimulant against chicken infectious bronchitis.

To study the microstructure of the internal organs, specifically, spleen and liver, the samples of the following size: $0.5 \times 1.5 \times 0.5 \mathrm{~cm}$, were taken after slaughter. The material was fixed in a $10 \%$ neutral formalin aqueous solution for three days. The fixed pieces of organs were dehydrated and treated with chloroform. The pieces of liver and spleen dehydrated in alcohol of increasing density were compacted by being poured in paraffin. Paraffin sections were made using paraffin blocks on a sledge microtome and were glued to a glass slide. After removing paraffin residues, the sections were clarified with carbol-xylene and treated with balsam. The preparations were coloured with hematoxylin-eosin. When studying the microstructural changes in the internal organs and tissues of chickens, the most pronounced changes were photographed on an MBI-6 microscope with a camera Pentax.

\section{Results}

On the $7^{\text {th }}$ day after vaccination with "Raspol" as an immunostimulant against chicken infectious bronchitis, the presence of numerous forming lymph nodes located near the central arteries is observed over the entire surface of the organ cuts. The peculiarities of the initial vaccination period include the weak manifestations of the connective tissue disorganization in the organ vessels walls. All of them retained the lumen. In addition, hemocirculation in the full-blooded capillary network was provided in the emerging lymphoid tissue of the nodules. Numerous forming germination centers consisted of reticulocytes, whose majority was transformed into dendritic forms, and blast, slightly differentiated cells. Compared with control chickens, the initial stages of the lymph nodes formation were more pronounced in the spleen and hemocirculation disorders were less pronounced in the small vessels of this organ.

On the $14^{\text {th }}$ day after vaccination, the histological structure of the organ was well marked. The numerous lymph nodes that were being formed had expanded periarterial zones saturated with small T-lymphocytes. The walls of the blood vessels (central arteries, capillaries), lymph nodes were dilated with marked profiles of the gaps. The germinal centers of the nodules became enlarged, containing the blast forms of cells, large lymphocytes and reticulocytes of the dendritic form. It should be noted that the profiles of capillaries newly formed near the breeding centers were well preserved. As a result of lymphoid tissue hyperplasia, the boundaries of the mantle and marginal zones became sharply marked. For this period, lymphoproliferative processes markedly increased in the spleen, combined with the absence of local hemodynamic disorders and disorganization of the main substance of the connective tissue.

On the $21^{\text {st }}$ day after vaccination, the formed lymph nodes with a pronounced structure of reactive centers were located on the surface of the organ cut. These centers were distinguished by an increased density of medium and small lymphocytes, which hided the presence of reticular cells in some places. Hyperplasia of the germination centers cells caused a sharp squeezing of the surrounding reticular base of the white pulp. The adjacent mantle zone of the nodules was even more saturated with lymphocytes and merged with a wide marginal region to the periphery without a clear boundary. Significant accumulations of small lymphocytes were clearly defined around the central arteries. Weak signs of their mucoid swelling should be considered as mild reactogenic effects of the vaccine, introduced together with the polysaccharide "Raspol". Endotheliocytes nuclei swelling, weak effects of the wall layers homogenization and weakly expressed perivascular edema were noted in them against the background of the lumen profiles preservation. The absence of edema zones around the central trabecular blood vessels of the organ should also be noted.

On the $28^{\text {th }}$ day after vaccination, the organ structure iwas well defined. Numerous reactive centers were located evenly on the surface of the organ cut. The cellular composition of these formations represented clusters with a lower density than the one noted at the previous research stage. Numerous reticular cells, large and medium lymphocytes were noted in the reactive centers, between which blast and poorly differentiated cells were designated, and vascular profiles were sometimes found in the cells. The previous hyperplasia of lymphoid tissue cells contributed to the contrasting designation of the structurally functional zones of the lymph nodes. The mantle zone consisting of numerous medium and small lymphocytes was well designated. In contrast to previous periods, the predominance of medium lymphocytes over small ones was noted in the periarterial zones. The lumen profiles of the central trabecular arteries were well defined, and there were no signs of the connective tissue disorganization in their walls. The marginal region of the lymph nodes also remained well defined, especially from the side of the inner border and consisted of a large number of small and fewer medium lymphocytes, while its width decreased markedly compared to the previous organ examination. The peripheral part of the marginal zone gradually merged with a moderate blood-filled red pulp. The indicated changes at this research stage noted the signs of lymphoid cell hyperplasia in the lymph nodes with weak or no consequences of the vaccine reactogenic effect on the organ connective tissue components.

On the $7^{\text {th }}$ day after vaccination with "Fosprenil" as an immunostimulant, the histological structure of the organ was pronounced. Numerous germinative centers of the lymph nodes under formation differed in size and cell density. Around the germinal centers, a thin layer of medium and small lymphocytes 3-4 cells large formed 
the mantle zone boundary. Most germination centers were small in area. Their vessels were dilated. The endothelial cells in the vessels were swollen, which narrowed the lumen. The cell composition of germination centers was represented mainly by dendritic cells and fewer blast cells, slightly differentiated cells and rare mitotic figures. The central arteries retained profiles of the lumen, and their walls remained little changed. The periarterial area of lymphocyte accumulation around these vessels was not pronounced. The moderate blood-filled red pulp of the organ contained a small amount of small and medium lymphocytes between the reticulocytes. By this time, germination centers maintained their value similar to the previous research stage. The cellular composition in them has changed towards lymphoid cells. Compared with reticular cells, the content of lymphoblasts and cell mitosis figures increased. In the germinal centers of nodules the vascular profiles of germinal centers were no more indicated due to an increase in the number of lymphocytes, and they continue to be detected only in individual nodules. The newly formed lymphoid tissue surrounding germinative centers significantly increased and generated lymphocyte zones uneven in thickness and density and indicating the internal boundaries of the mantle zone. The outer borders of this zone without any visible outlines, merged with the surrounding moderate blood-filled red pulp.

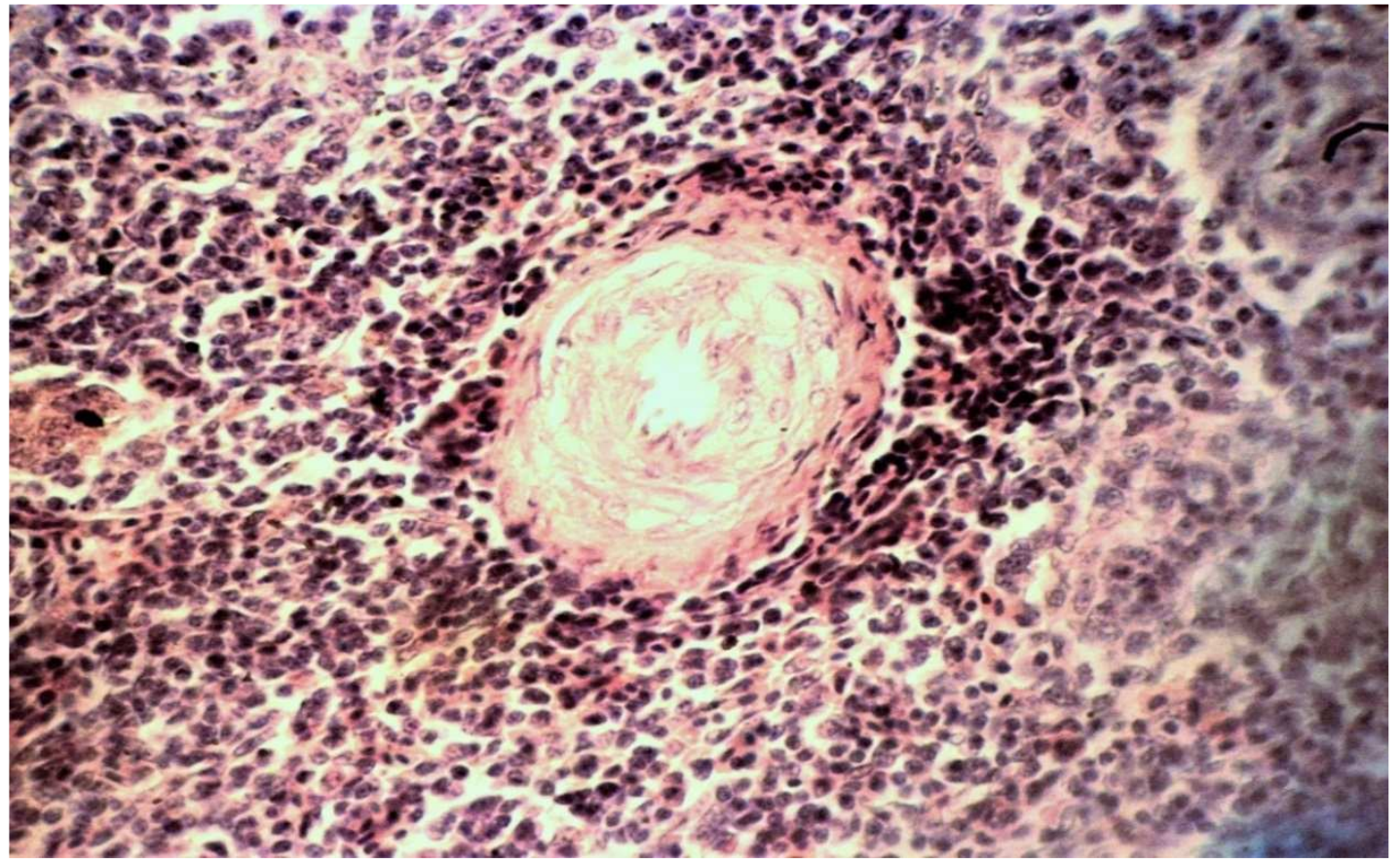

Fig. 1. Chicken spleen on the $28^{\text {th }}$ day after vaccination with "Raspol". Hematoxylin and eosin stain. X 400

By the $14^{\text {th }}$ day, germination centers retained their value similar to the previous research stage. Their cellular composition has changed towards lymphoid cells. Compared with reticular cells, the content of lymphoblasts and cell mitosis figures increased.

On the $21^{\text {st }}$ day, the histological structure of the organ became moderately pronounced. The germinal centers of the lymph nodes did not change in size. They markedly decreased the density of cells and their composition still consisted of blast forms of cells, reticulocytes, and less numerous medium and small lymphocytes. Figures of mitosis were observed only in individual germination centers. The mantle zone became noticeably thinner. Its borders did not enlarge. It merged with the surrounding red pulp due to not pronounced boundaries. The nodules periarterial zones were also sparse. A sparse T-dependent zone was formed in it in separate sections being 15-20 small lymphocytes large. The walls of the central arteries were swollen. The nuclei of the endotheliocytes swelled into the lumen of the vessels and, as a result, the lumens profiles were narrowed. Due to moderate hyperplasia of the germinal centers cells, gaps of small vessels continued to be detected in them.
Most lymph nodes had not increased by the end of the experiment, and only individual nodes slightly expanded their area. They markedly increased the content of medium and large lymphocytes. However, the blast forms of cells and figures of mitosis became significantly less than they had been at earlier research stages. The mantle zone surrounding germinative centers remained thinned and was distinguished by a thin layer of small lymphocytes, consisting of 7-8 layers of cells. Due to the lower level of cells proliferative activity and, consequently, the lower density of the newly formed cells location in the germinal centers, the profiles of the lumen of small vessels were indicated. The signs of mild mucoid swelling were noted in the walls of the trabeculae vessels.

In birds of the second group, the stimulation of lymphoproliferative activity continued for the first two weeks after vaccination and by the end of a one-month period it was no longer detected, while the signs of moderate post-vaccination disorganization of the connective tissue components were detected during the whole period of observation. 


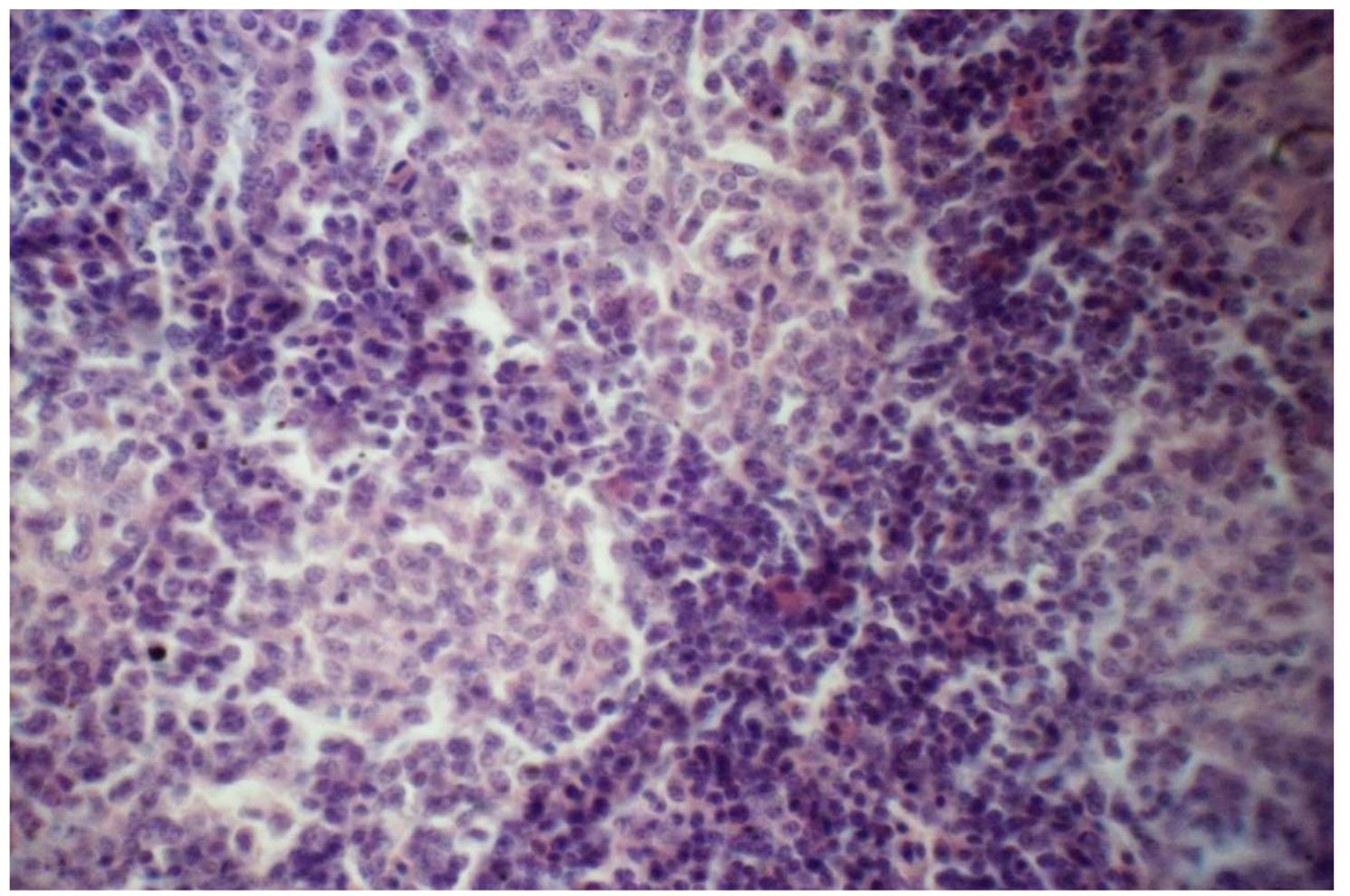

Fig. 2. Chicken spleen on the $28^{\text {th }}$ day after vaccination with immunostimulator Fosprenil. Hematoxylin and eosin stain. X 400 .

The histological pattern of the organ of the control group on the $7^{\text {th }}$ day was moderately pronounced. No formed lymph nodes were detected on the entire surface of the organ cut at this research stage. Lymphoid tissue concentrated primarily around the central arteries of the organ. Single germinative centers whose boundaries were indicated by squeezed, swollen processes of reticulocytes and the mantle zone surrounding them by lymphocytes were sometimes found in the organ cut. Single rare blast forms of cells were also located in sparse germination centers under formation. In small, moderately filled blood vessels of the organ, the structure of the wall layers was preserved. Focal sparse accumulations of lymphocytes were distinguished in a weakly blood-filled red pulp of an organ against the background of reticulocytes with thickened processes. Consequently, proliferative processes with a predominant concentration of small lymphocytes in the periarterial zones were only beginning to appear in the spleen.

On the 14th day, small-sized lymph nodes with a moderate designation of structural and functional zones were evenly located in the organ cuts. By the time of the study, small germinal centers consisting of reticulocytes with thickened processes, single figures of the mitosis of cells, lymphoblasts, and medium lymphocytes arose in the lymph nodes. The greatest cells concentration was noted in the periarterial one. Mantle and marginal zones of nodules were poorly marked due to the lymphocytes low density. The lumens of the central arteries were sharply narrowed as a result of the endothelial cells swelling as well as media and adventitia swelling and homogenization. Mucoid swelling of the central arteries walls was combined with perivascular edema.
The next research stage marked the manifestations of previous hyperplasia of lymphoid tissue, which were noted in the spleen. The oval-shaped lymph nodes became large. The density of lymphocytes greatly increased in the periarterial zone and the boundaries of the mantle zone got more sharply marked. At the same time, the germinal zone and the marginal area border were poorly marked, which indicated the lymphoid tissue hyperplasia in the white pulp caused by previous antigenic stimuli. In most vessels, signs of mucoid swelling of perivascular edema were noticeably weakened. In the moderate blood-filled red pulp of the organ, the reticular cells retained thickened processes, between which lymphocytes were located sparsely.

On the $28^{\text {th }}$ day the organ showed the maximum degree of lymphoid tissue hyperplasia compared with the previous research stages. Enlarged lymph nodes with unclear borders were located separately. Lymph nodes had expanded germinal centers, but a sparse cellular composition. Among the numerous reticulocytes with swollen processes, mainly small and medium lymphocytes and single blast cells were located in these centers. At the same time, mitosis figures were not determined. The boundaries of the structural and functional zones of all lymph nodes were erased due to low cell density. In a weak blood-filled red pulp, sparse compact and diffuse accumulations of small lymphocytes were also detected among the reticulocytes. Blood vessels were distinguished by moderate severity of mucoid walls swelling, lumen profiles narrowing and the endotheliocytes pycnomorphic nuclei swelling. Having become small, the periarterial zones of the nodules lymphoid tissue were also noticeably sparse.

28 days after vaccination, lymphoproliferative processes noticeably weakened in the spleen of the 
control group of birds while the signs accompanied by moderate disorganization of the connective tissue components were kept.

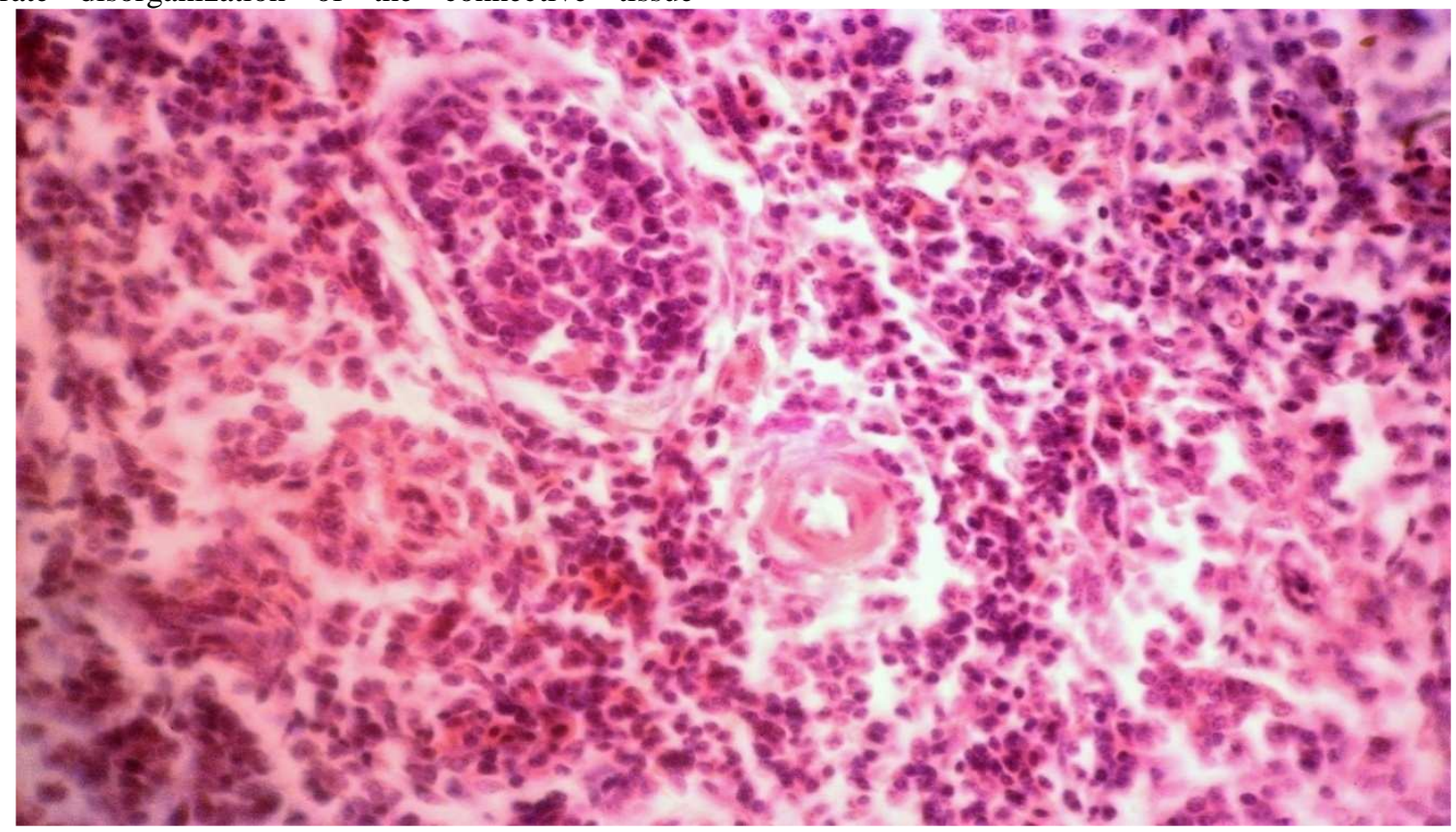

Fig. 3. Histological pattern of spleen of the chicken from the control group on the $28^{\text {th }}$ day after vaccination. Hematoxylin and eosin stain. X 200.

\section{Conclusion}

Thus, the studied physiological, morphological, biochemical, immunological parameters of the chickens blood and histological studies make it possible to recommend the use of the heteropolysaccharide Raspol as an immunostimulant for vaccinating young birds against infectious bronchitis.

\section{References}

1. V. Fisinin, N. Zhuravel, A. Miftakhutdinov, Veter. Med. 6.14 (2018)

2. G. Bobyleva, Poultry and poultry prod. 3, 22 (2017)

3. N. Kuzmenko, A. Kuzmenko, A. Krakosevich, A. Gnenko, Livestock of Russia 5, 18-19 (2017)
4. R. Asrutdinova, Sci. notes of the Kazan State Acad. of Veter. Med. named after N.E. Bauman 211, 214 218 (2012)

5. I. Barinsky, A. Lazarenko, L. Alimbarova, Immunol. 4, 181 (2012)

6. M. Zadorozhnaya, The effect of betulin on the immune system of chickens during vaccinations, Poultry farm. 4, 61 (2011)

7. K. Zimin, Poultry and poultry prod. 2, 50 (2016)

8. M. Kamalieva, R. Asrutdinova, S. Garipov, Bull. of the Krasnoyarsk State Agrar. Univer. 5, 35-39 (2017)

9. E. Ovseychik, Growing broiler chickens using immunomodulators, Poultry 11-12, 41 (2018)

10. E. Voronin, A. Petrov, M. Serykh, Immunology (Kolos-press, Moscow, 2002), 406 p.

11. Y. Gao et al., J. Med. Food 8(2), 159-168 (2005) 